\title{
BMJ Open Cross-sectional study of the provision of interventional oncology services in the UK
}

\author{
Jim Zhong, ${ }^{1}$ Peter Atiiga, ${ }^{1}$ Des J Alcorn, ${ }^{2}$ David Kay, ${ }^{2}$ Rowland Illing, ${ }^{3}$ \\ David J Breen, ${ }^{4}$ Nicholas Railton, ${ }^{5}$ Ian J McCafferty, ${ }^{6}$ Philip J Haslam, ${ }^{7}$ \\ Tze Min Wah ${ }^{1}$
}

To cite: Zhong J, Atiiga P, Alcorn DJ, et al. Crosssectional study of the provision of interventional oncology services in the UK. BMJ Open 2017;7:e016631. doi:10.1136/ bmjopen-2017-016631

- Prepublication history and additional material for this paper are available online. To view please visit the journal (http:// dx.doi.org/10.1136/bmjopen2017-016631).

Received 18 April 2017 Revised 18 July 2017 Accepted 27 July 2017

\section{CrossMark}

${ }^{1}$ Department of Clinical and Interventional Radiology, Institute of Oncology, St James's University Hospital, Leeds, UK ${ }^{2}$ Department of Interventional Radiology, Gartnavel General Hospital, Glasgow, UK ${ }^{3}$ Department of Interventional Radiology, University College Hospital, London, UK

${ }^{4}$ Department of Radiology, Southampton University Hospitals, Southampton, UK ${ }^{5}$ Department of Interventional Radiology, Mid Essex Hospital Services NHS Trust, Chelmsford, UK

${ }^{6}$ Department of Radiology, Queen Elizabeth Hospital Birmingham, University Hospitals Birmingham NHS Trust, Birmingham, UK ${ }^{7}$ Department of Interventional Radiology, Freeman Hospital, Newcastle-upon-Tyne, UK

Correspondence to Dr Tze Min Wah; tze.wah@nhs.net

\section{ABSTRACT}

Objective To map out the current provision of interventional oncology (I0) services in the UK. Design Cross-sectional multicentre study.

Setting All National Health Service (NHS) trusts in England and Scottish, Welsh and Northern Ireland health boards. Participants Interventional radiology (IR) departments in all NHS trusts/health boards in the UK.

Results A total of 179 NHS trusts/health boards were contacted. We received a $100 \%$ response rate. Only $19(11 \%)$ institutions had an 10 lead. 144 trusts $(80 \%)$ provided 10 services or had a formal pathway of referral in place for patients to a recipient trust. 21 trusts $(12 \%)$ had plans to provide an 10 service or formal referral pathway in the next 12 months only. 14 trusts $(8 \%)$ did not have a pathway of referral and no plans to implement one. 70 trusts (39\%) offered supportive and disease-modifying procedures. One trust had a formal referral pathway for supportive procedures. 73 trusts (41\%) provided only supportive procedures (diagnostic or therapeutic). Of these, $43(59 \%)$ had a referral pathway for disease-modifying 10 procedures, either from a regional cancer network or through IR networks and 30 trusts $(41 \%)$ did not have a referral pathway for disease-modifying procedures. Conclusion The provision of 10 services in the UK is promising; however, collaborative networks are necessary to ensure disease-modifying 10 procedures are made accessible to all patients and to facilitate larger registry data for research with commissioning of new services.

\section{INTRODUCTION}

More than one in three people will develop cancer in their lifetime. ${ }^{1}$ Since the National Health Service (NHS) Cancer Plan in 2000, the Department of Health has strived to improve diagnosis and treatment. ${ }^{1}$ As part of the NHS Cancer Plan to deliver world-class cancer services, there is a drive to achieve better outcomes by ensuring all patients have access to the best treatments available. ${ }^{2}$ Wide variation remains in performance across the country with major differences in access to cancer services. ${ }^{1}$

Interventional oncology (IO) the use of image-guided techniques to diagnose and treat
Strengths and limitations of this study

This is the first study to investigate the provision of interventional oncology services in the UK.

- The sample size is large and covers all acute trusts and health boards in the UK.

- Cross-sectional study design allowed for multiple variables to be studied.

- Data were self-reported and therefore at risk of incompleteness.

- Limitations include those inherent to survey/ questionnaire format, including subjective bias.

patients with cancer is fast becoming the fourth pillar of oncological care alongside medical, surgical and radiation oncology; the Royal College of Radiologists have set out best practice guidance for the incorporation of IO into all cancer services nationally. ${ }^{3}$

Supportive and symptomatic procedures were defined as those providing relief from tumour-related symptoms, but not modifying the underlying malignant disease process and include diagnostic tests such as imageguided biopsy which 'support' the provision of definitive treatment. ${ }^{3}$ These may be palliative procedures such as image-guided drainage or stent insertion. Disease-modifying procedures were defined as those where the intent is to modify malignant progression and/or modify the prognosis and include image-guided ablation, transarterial chemoembolisation (TACE) and selective internal radiation therapy (SIRT).

There remains a significant shortage of interventional radiologists, who are the primary contributors towards IO, with almost half of services in England unable to provide a local or networked out of hours access to interventional radiology (IR). ${ }^{4}$ Undoubtedly, this shortfall will have consequences on the provision of elective IO services in the UK 
and potentially affect patient care through limitations to access.

The current provision of IO services throughout the UK is unknown; therefore, NHS commissioners are unable to realistically factor IO into national cancer pathways as evident in a previous Department of Health publication, ${ }^{1}$ which did not acknowledge IO as a treatment option for patients.

The primary objective of this cross-sectional study was to map out the current provision of IO services in the UK. We also sought to uncover formal patient referral pathways, the types of IO procedures offered and any limitations to providing IO. Ultimately, we aim to develop IO networks and improve access to these treatments for patients with cancer.

The survey was designed and undertaken by theInterventional Oncology United Kingdom (IOUK) group, a specialist interest group of the British Society of Interventional Radiology(BSIR).

\section{METHODS}

No research ethics committee approval was required for this data-gathering project. No patient identifiable data was captured.

This cross-sectional study involved all acute NHS Foundation Trusts in England ${ }^{5}$ and all health boards in Scotland, Wales and Northern Ireland which were contacted via email with the survey (see online supplementary material). This was followed by a telephone follow-up of all hospital radiology departments that did not complete the

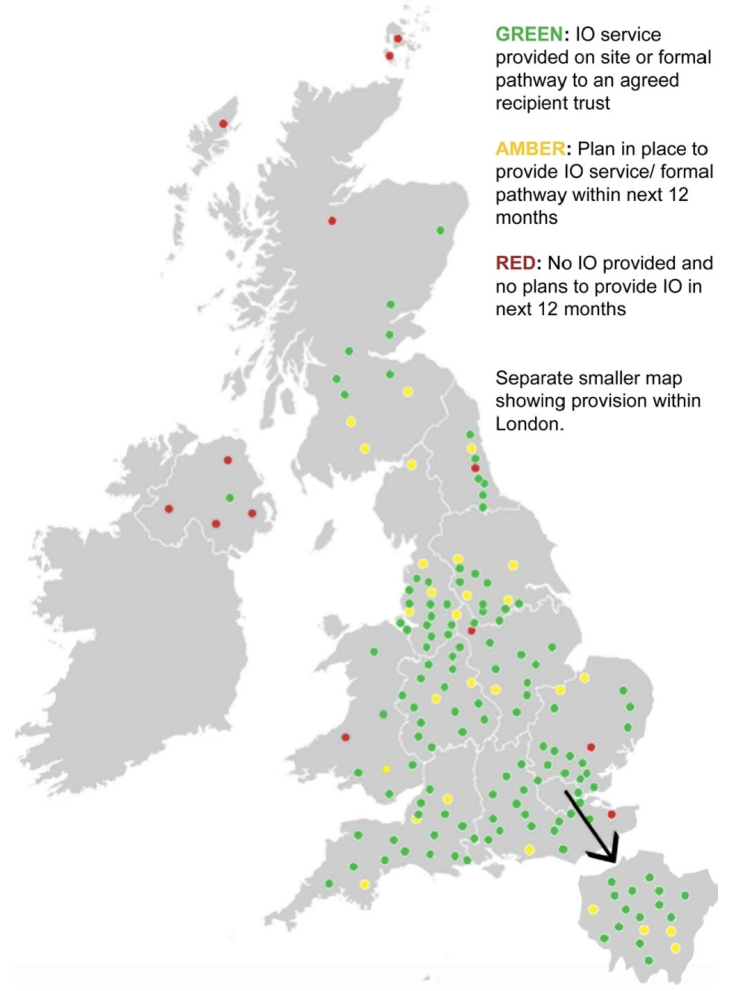

Figure 1 UK map showing the overall provision of interventional oncology $(\mathrm{IO})$ services throughout the UK. survey within 2 weeks of the first email being sent out. Telephone follow-up was conducted by a single radiologist (JZ). The survey could be completed by any of the following: The head of department of radiology/IR, any consultant radiologist (diagnostic or interventional) or superintendent radiographer who has insight into the local provision of services.

The surveys key points were the following:

- Are IO procedures offered in the trust?

- If so, are these supportive treatments only or both supportive and disease modifying?

- We asked about the types of procedures undertaken.

- If no IO procedures are offered, is there an agreed formal pathway to another recipient trust?

- If there was no agreed pathway, was there a plan to provide IO or a pathway in the next 12 months?

- What barriers are there to setting up an IO service?

The full survey can be found in the online supplementary material.

Following the initial survey, we followed up all trusts/ health boards which only offered supportive treatments to see if there were formal referral pathways for disease-modifying procedures.

A formal referral pathway was defined as an existing mechanism of referral through a multidisciplinary team responsible for the patient, usually through a pre-existing local oncology or radiology network.

\section{RESULTS}

A total of 179 NHS trusts or health boards were contacted throughout the UK. We received $100 \%$ response rate. The responses came from consultant interventional and diagnostic radiologists and superintendent radiographers who had insight into the local provision of services.

One hudred and forty-three trusts $(80 \%)$ had an IR department in their trust. All trusts with an IR department offered IO procedures. Only 19 (11\%) institutions had an IO lead.

One hundred and forty-four trusts $(80 \%)$ provided IO services or had a formal pathway of referral in place for patients to a recipient trust (figures 1 and 2), of which 137 trusts (77\%) stated what types of IO services they offered. Twenty-one trusts (12\%) had plans to provide an IO service or formal referral pathway in the next 12 months only. Fourteen trusts (8\%) did not have a pathway of referral and no plans to implement one.

Seventy trusts (39\%) offered both supportive and disease-modifying procedures (figure 3). One trust had a formal referral pathway for supportive procedures. Seventy-three trusts $(41 \%)$ provided only supportive procedures (diagnostic or therapeutic).

The number of trusts providing each supportive/symptomatic IO procedure and disease-modifying procedure are shown in table 1 and table 2, respectively. The seven trusts that gave no details to which IO procedures were offered were excluded when calculating the percentages. 


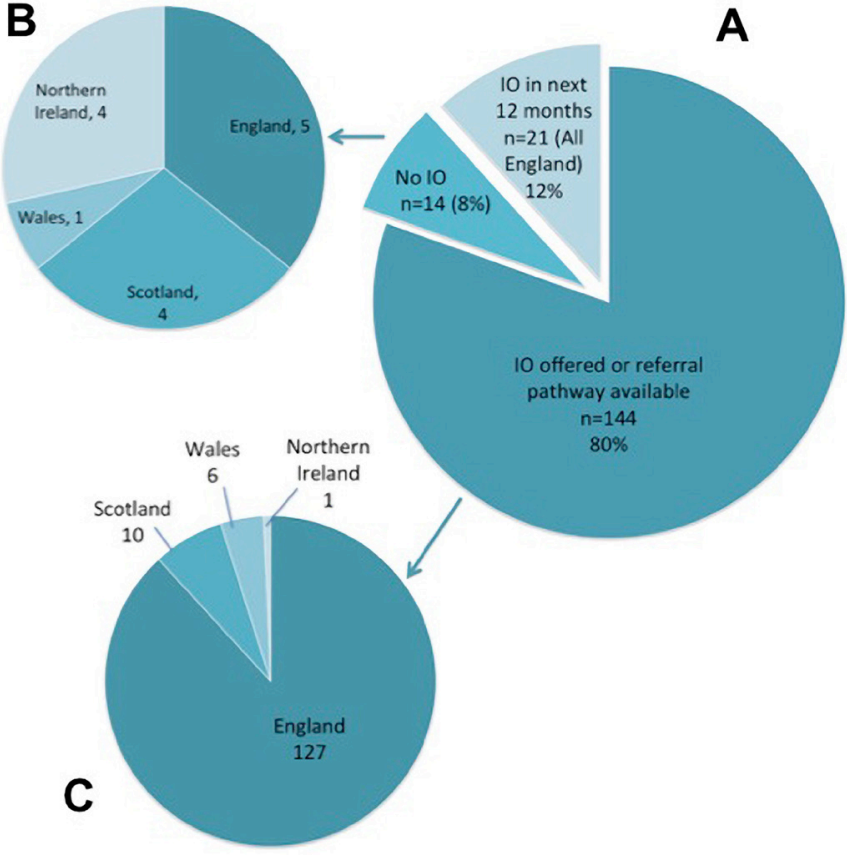

Figure 2 Middle pie chart (A) displaying total number/ percentage of trusts which offer interventional oncology (IO) or have a referral pathway, number which plan to set up IO service or referral pathway in the next 12 months (all English trusts) and those without any plans to set up an IO service pathway. The top $(\mathrm{B})$ and bottom $(\mathrm{C})$ pie charts display the breakdown of healthcare trusts/health boards by country.

Out of 179 (72\%), 129 trusts offered therapeutic IO procedures after excluding trusts, which only offered diagnostic image-guided biopsy. Figures 4 and 5 show the maps of the trusts providing each type of disease-modifying procedure.

For England only, a total of 153 NHS Foundation trusts in England were contacted. One hundred and twenty-seven $(83 \%)$ trusts provided IO services or had a formal pathway of referral to another agreed recipient trust for IO procedures (figure 1). Out of the 26 that did not have a formal referral pathway, $21(14 \%)$ trusts had plans to provide an IO service or formal referral pathway for patients to have IO at another trust within the next 12 months. Five (3\%) trusts did not have a pathway and had no plans of providing IO or a referral pathway in the next 12 months (figure 1). Out of 127 (45\%), 57 trusts providing IO offered both supportive and disease-modifying procedures (figure 3). Out of 127 (55\%), 70 provided only supportive procedures.

For Scotland, 10 out of 14 health boards provided IO or had a formal pathway of referral to a specialist hospital in another health board (Glasgow or Edinburgh). Of these, eight health boards provided both supportive and disease-modifying IO, while two provided only supportive IO. Four health boards (29\%) did not provide IO and did not have plans to provide a pathway in the next 12 months.

For Wales, six out of seven health boards (86\%) provided IO or had a referral pathway in place. Four Welsh health boards (57\%) provided both types of IO,

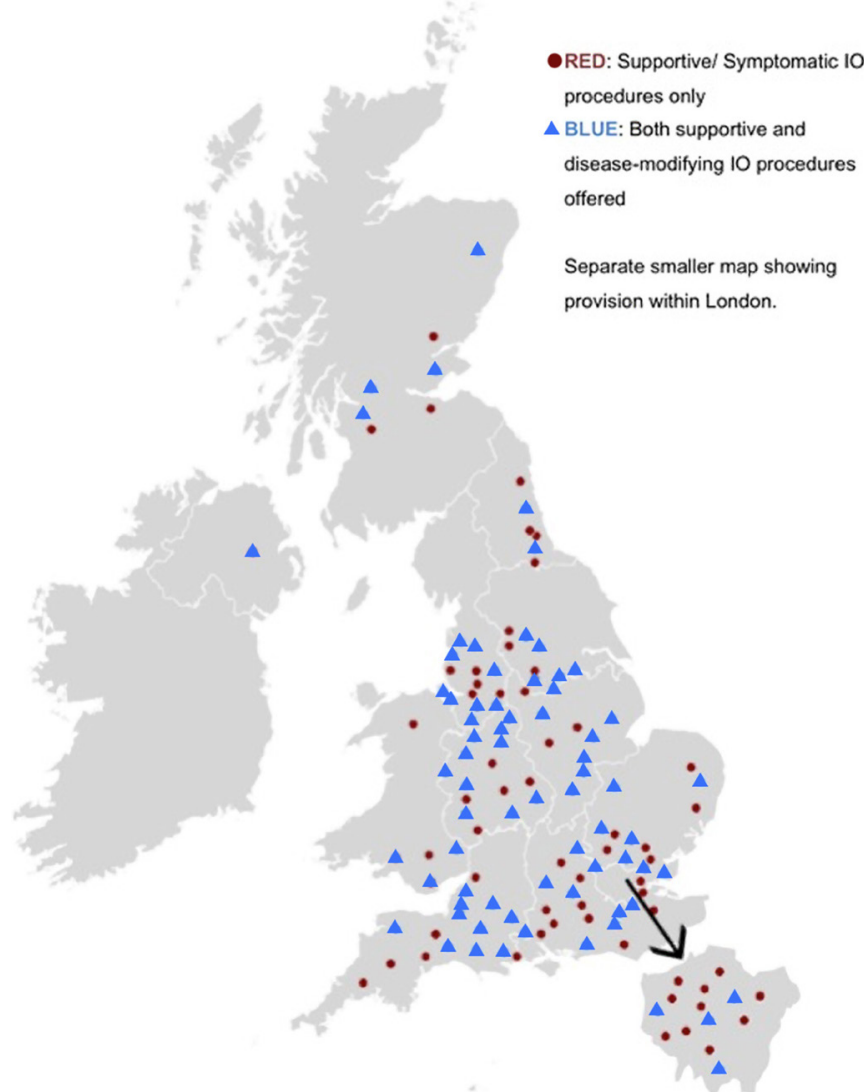

Figure 3 UK map showing what types of interventional oncology (IO) procedures (supportive and/or diseasemodifying procedures) are undertaken in each National Health Service trust/health board.

one health board provided only supportive IO and one had a formal referral pathway. One Welsh health board did not offer IO or have a referral pathway implemented in the next 12 months.

Only one out of five health boards in Northern Ireland (Belfast Health and Social Care Trust) provided IO (both types). Four health boards did not have plans to offer IO or have a referral pathway implemented in the next 12 months.

Out of the 70 English NHS trusts and three Scottish/ Welsh Health Boards, which provided only supportive IO, 43 trusts $(59 \%)$ had a referral pathway to another hospital/trust for disease-modifying IO procedures. This was from a local regional cancer network referral initiated following a formal discussion at the multidisciplinary team meeting or through IR networks. Thirty trusts $(41 \%)$ did not have a referral pathway for disease-modifying procedures.

The most common barriers to providing disease-modifying IO were insufficient funding, lack of staff, lack of support from other non-radiology clinicians, having a pathway already in place and problems with recruitment into IR. 
Table 1 Type of supportive/symptomatic IO procedure and number of trusts that offer each and percentage of total number of trusts that provided information on the types of procedure offered $(n=137)$

\begin{tabular}{llc}
\hline $\begin{array}{l}\text { Type of supportive/symptomatic IO } \\
\text { procedure }\end{array}$ & $\begin{array}{l}\text { No of } \\
\text { trusts }\end{array}$ & $\begin{array}{l}\text { Percentage } \\
\text { of total }\end{array}$ \\
\hline Image-guided biopsy & 137 & 100 \\
\hline Nephrostomy & 129 & 94 \\
\hline Image-guided drainage & 128 & 93 \\
\hline Central venous catheter & 125 & 91 \\
Ureteric stenting & 124 & 91 \\
Biliary drainage and stenting & 118 & 86 \\
Vena caval filtration & 88 & 64 \\
Gastrointestinal stenting & 87 & 64 \\
Percutaneous transhepatic & & \\
cholangiography & 84 & 61 \\
Enteral tube placement, for example, & & \\
radiologically inserted gastrostomy & 81 & 59 \\
\hline Vena caval stenting & 79 & 58 \\
\hline Ascitic diversion & 39 & 28 \\
\hline Vertebroplasty & 6 & 4 \\
\hline Isolated perfusion chemotherapy & 5 & 4 \\
\hline
\end{tabular}

Out of 144,7 who offered IO did not include what procedures were offered and were excluded from calculations.

$\mathrm{IO}$, interventional oncology.

\section{DISCUSSION}

Overall, the provision of IO in the UK is unevenly spread. Based on the Royal College of Radiologists definition of 'supportive' IO, ${ }^{1}$ this encompasses many routine procedures that can be carried out by diagnostic radiologists which is reflected in the excellent availability of these procedures throughout the UK. Beyond basic image-guided drainage procedures, the provision of specialist vascular, gastrointestinal or biliary 'symptomatic' intervention is less routinely available apart from in the larger tertiary centres which were also providing

Table 2 Type of disease-modifying 10 procedures and no of trusts that offer each and percentage of total no of trusts $(n=179)$

\begin{tabular}{lll}
\hline $\begin{array}{l}\text { Type of disease-modifying } 10 \\
\text { procedure }\end{array}$ & $\begin{array}{l}\text { No of } \\
\text { trusts }\end{array}$ & Percentage \\
\hline Transarterial chemoembolisation & 40 & 22 \\
\hline Liver ablation & 39 & 22 \\
\hline Kidney ablation & 39 & 22 \\
\hline Lung ablation & 28 & 16 \\
\hline Bone ablation & 18 & 10 \\
\hline Selective internal radiation therapy & 17 & 9 \\
\hline Prostate ablation & 2 & 1 \\
\hline
\end{tabular}

$\mathrm{IO}$, interventional oncology.

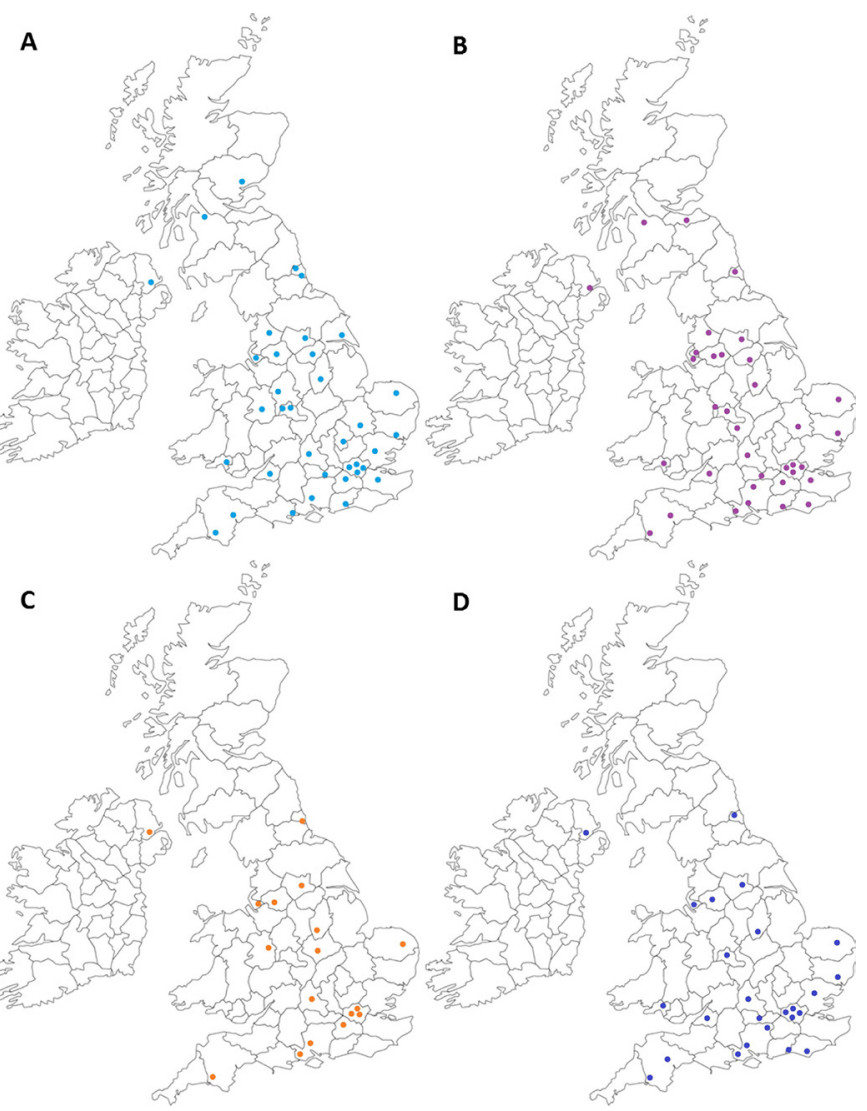

Figure 4 UK map showing the provision of ablation services: (A) renal ablation, (B) liver ablation, (C) bone ablation and (D) lung ablation.

disease-modifying IO. This highlights important areas to target nationally for radiology and IO training.

Given the vital role of IR in the management of critically ill patients, the comprehensive provision of supportive IO in most parts of the UK reflects the drive to train more radiologists with basic interventional skills, which are also transferrable to IO. ${ }^{6}$ Only one trust without an IR department offered IO. We were unable to capture if most of the IO procedures were done by the IR department or not, with institutions occasionally splitting non-vascular (eg,

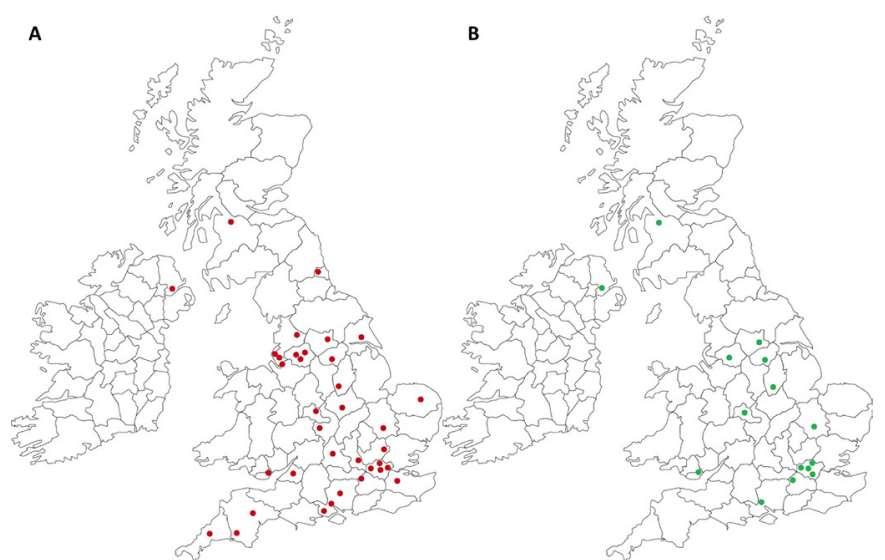

Figure 5 UK map showing the provision of $(A)$ transarterial chemoembolisation and $(B)$ selective internal radiation therapy. 
ablation) and vascular interventions (eg, TACE) between the diagnostic radiologists and the interventional radiologists who also have to cover the on-call service for non-oncology-related emergency procedures such as trauma, bleeding or aortic syndromes. A major recruitment drive currently is the provision of on-call IR services and given the overlap between IO and IR training, emergency IR provision is therefore linked with the provision of IO services not only for maintaining the availability of supportive services in small district hospitals, but also for the provision of disease-modifying IO in specialist centres. Additional need for interventional radiographers and nursing cover for IO services should not be overlooked either to allow a new IO service to be introduced.

We acknowledge that disease-modifying treatments form a smaller proportion of IO workload and centralisation of this is happening as part of the current NHS model for cancer services. ${ }^{7}$ One argument for this model in the context of IO is to ensure that more complex IO procedures are undertaken by those who carry out a sufficient number of cases to maintain competency; however, this should not preclude suitable patients from being referred due to their geographical location. There are NHS trusts/boards, most noticeably in rural Scotland and Northern Ireland where access to disease-modifying IO services appears limited and linking up with neighbouring hospitals to set up formal referral pathways should be considered. What also remains unclear is what the current demand for IO services are generally, but particularly in these rural regions, as we have no data to suggest current arrangements are suboptimal. It would not be necessary or appropriate for all providers to liaise with IO services, and these should be facilitated through regional cancer networks with more integrated pathways of care. ${ }^{7}$ Currently there appears to be 136 out of 153 acute NHS trusts in England, which are listed to offer acute oncology services. ${ }^{8}$ Further work is required to elucidate whether there is any discrepancy in the regional demand and supply of disease-modifying IO.

The perceived barriers to starting up elective disease-modifying services stemmed from shortfalls in funding, staffing and support from other specialties. With tertiary centres undertaking much higher volumes of disease-modifying IO procedures, some smaller district general hospitals felt unsupported in starting up their own service, from financial considerations when purchasing the equipment to garnering support from allied specialties such as surgery and oncology. This is an important point as it highlights the need for greater awareness of the role of the interventional radiologist in oncology care and we must strive to work even closer with oncologists given the new evidence suggesting the added value of combination therapies and incorporation of IO procedures into European cancer guidelines. ${ }^{9}$ However, with the current model of centralising cancer services, these barriers would only be an issue if cancer centres were unable to provide IO. To improve patient selection for complex IO procedures, interventional radiologists should have a regular role in multidisciplinary team meetings. With only 19 institutions (11\%) currently having a formal IO lead clinician, there is a role for dispersed leadership to achieve structural change in established cancer networks.

Local expertise and facilities help determine the IO that is offered. An example is the provision of disease-modifying $\mathrm{IO}$ for the liver, which is centred around the national liver transplant units. ${ }^{10}$ Clearly image-guided tumour ablation, TACE and SIRT are effective therapies than can be used solely or in combination with chemotherapy or surgery to improve the outcome of such patients. ${ }^{11} 12$ Participation in MDT discussions will also allow interventional radiologists and radiologists familiar with IO techniques to educate other clinicians on the role of IO in the management of patients and contribute towards improvement and restructuring of services. This will also open opportunities to undertake collaborative research that will be higher impact and wider reaching to the oncology community.

The recurrent issue of lack of staffing within IR remains a barrier. Despite the promising provision of supportive IO, most departments are struggling to cope with the demand for basic vascular, urological and biliary procedures, necessary to provide a sustainable out of hours service, without compounding this with additional workload and need for additional training for disease-modifying IO.

Changes to the delivery of healthcare throughout the UK demands that IO treatments can demonstrate not only a therapeutic benefit but also cost-effectiveness. For units with a referral pathway for disease-modifying IO, there was a common theme that this set-up was more cost-effective than starting a service from scratch. Without knowledge of the actual demand for these IO procedures, there is no answer to this currently, and clinical investigators must incorporate measures of cost-effectiveness and patient-reported outcomes into large-scale studies to provide more robust evidence. ${ }^{13}$ Even if these IO treatments can be shown to be equally effective compared with the current standard of care but with significantly less morbidity, then it will allow the specialty to develop further; however, current studies have not offered definitive conclusions. ${ }^{14}$ Building on the knowledge of these IO networks will allow better registry data that can be used to derive larger cohorts for future trials and also commissioning of new services.

The limitations of the present study include those inherent in the survey/questionnaire format such as the subjective element depending on whether a superintendent radiographer or consultant radiologist responded given their underlying experience and knowledge of their radiology services which could impact on the detail of their survey answers. The strengths of the survey include $100 \%$ response rate from 179 acute NHS trusts/health boards which allowed a comprehensive map of both supportive and disease-modifying IO procedures offered in the UK that will help direct radiology/IO training, 
future planning of new IO services and allow for more integrated cancer pathways.

\section{CONCLUSION}

The provision of IO services in the UK is promising; however, collaboration and networking is necessary to ensure disease-modifying IO procedures are made accessible to all patients throughout the UK and to facilitate improved registry data collection for research and commissioning or funding of new services.

Acknowledgements We thank AngioDynamics for funding the open access publication fee of this study.

Contributors JZ was also involved with designing the initial study concept, initial literature search, designing the survey and questionnaire layout, all data collection and analysis, writing up the manuscript and revising the manuscript in discussion with other authors. He has prepared the manuscript for publication. PA was also involved with designing the survey and questionnaire layout, the data collection and revising the manuscript in discussion with other authors. DJA, DK, RI, DJB, NR, IJM, PJH were also involved with finalising the initial study concept, reviewing and approving the survey and questionnaire layout, reviewing the data and analysis and approving the manuscript for publication in discussion with the other authors. TMW was also involved with designing the initial study concept, approving the survey and questionnaire layout, reviewing the results and analysis and revising the manuscript in discussion with other authors and approving the manuscript for publication in discussion with the other authors.

Funding AngioDynamics funded the open access publication fee of this study. Competing interests None declared.

Provenance and peer review Not commissioned; externally peer reviewed. Data sharing statement № additional data are available.

Author note DJA, DK, RI, DJB, NR, IJM, PJH, TMW are members of the Interventional Oncology United Kingdom (IOUK) group, a specialist interest group of the British Society of Interventional Radiology (BSIR).

Open Access This is an Open Access article distributed in accordance with the Creative Commons Attribution Non Commercial (CC BY-NC 4.0) license, which permits others to distribute, remix, adapt, build upon this work non-commercially, and license their derivative works on different terms, provided the original work is properly cited and the use is non-commercial. See: http://creativecommons.org/ licenses/by-nc/4.0/

(C) Article author(s) (or their employer(s) unless otherwise stated in the text of the article) 2017. All rights reserved. No commercial use is permitted unless otherwise expressly granted.

\section{REFERENCES}

1. NHS England Department of Health and Public Health England. Progress in improving cancer services and outcomes in England. 2015.

2. NHS England. Achieving world-class cancer outcomes: taking the strategy forward. 2016.

3. Royal College of Radiologists. Interventional oncology: guidance for service delivery. 2013 http://www.rcr.ac.uk/sites/default/files/ publication/RCR\%2813\%29_IO_0.pdf (accessed 6 Jun 2016).

4. Centre for Workforce Intelligence. Securing the future workforce supply: clinical radiology stocktake. London: Centre for Workforce Intelligence, 2012.

5. UK Government. Transparency data NHS foundation trust directory. 2015. https://http://www.gov.uk/government/publications/nhsfoundation-trust-directory/nhs-foundation-trust-directory (accessed 6 Jun 2016).

6. Royal College of Radiologists. Provision of Interventional Radiology Services. 2013 https://http://www.rcr.ac.uk/system/files/publication/ field_publication_files/BFCR(14)12_POIR.pdf (accessed 6 Jun 2016).

7. Independent Cancer Taskforce Cancer Research UK. Achieving world-class cancer outcomes, a strategy for England 2015-2020. 2015.

8. National Peer Review Programme. My cancer treatment. 2016 http:// www.mycancertreatment.nhs.uk/find-your-treatment/ (accessed 6 Jun 2016).

9. Escudier B, Porta C, Schmidinger M, et al. Renal cell carcinoma: ESMO clinical practice guidelines for diagnosis, treatment and follow-up. Ann Oncol 2016;27(suppl 5):v58-v68.

10. NHS Blood and Transplant. Transplant units in the UK. Secondary transplant units in the UK. 2016 http://www.odt.nhs.uk/ transplantation/transplant-units-in-uk/ (accessed 6thJune 2016).

11. Hickey R, Vouche M, Sze DY, et al. Cancer concepts and principles: primer for the interventional oncologist-part I. J Vasc Interv Radiol 2013;24:1157-64.

12. Hickey R, Vouche M, Sze DY, et al. Cancer concepts and principles: primer for the interventional oncologist-part II. J Vasc Interv Radiol 2013;24:1167-88.

13. Franklin JM, Gebski V, Poston GJ, et al. Clinical trials of interventional oncology-moving from efficacy to outcomes. Nat Rev Clin Oncol 2015;12:93-104.

14. Ljungberg $\mathrm{B}$, Bensalah $\mathrm{K}$, Canfield $\mathrm{S}$, et al. EAU guidelines on renal cell carcinoma: 2014 update. Eur Urol 2015;67:913-24. 